\title{
ASSOCIAÇÃO ENTRE INGESTÃO ALIMENTAR E RISCO DE SARCOPENIA EM PACIENTES IDOSOS EM HEMODIÁLISE
}

Larissa Sarti Lourenço, Bianca Barbosa De Farias, Leticia De Lima Oliveira, Sabrina Alves Lenquiste, Rayana Loch Gomes

Universidade do Oeste Paulista - UNOESTE, Presidente Prudente, SP. E-mail: rayana.loch@hotmail.com

\section{RESUMO}

Verificou-se a associação entre ingestão alimentar e risco de sarcopenia em pacientes idosos com doença renal crônica (DRC) em hemodiálise. Foram recrutados pacientes idosos, de ambos os sexos, do setor de hemodiálise de dois hospitais do interior paulista. Para compor o grupo, todos os indivíduos deveriam possuir diagnóstico de DRC e realizar hemodiálise. Os participantes $(n=25)$ tiveram suas medidas antropométricas avaliadas, seguida da aplicação do questionário SARC-F + circunferência da panturrilha (CP) para identificação do risco de sarcopenia. Ainda, os pacientes responderam a um diário alimentar para avaliação da ingestão alimentar. Foram encontradas associações entre ingestão diária de calorias, carboidratos, fósforo, ferro, sódio e valores do SARC-F+CP. Conclui-se que houve associação entre alguns fatores da ingestão alimentar e SARC-F+CP, de maneira que quanto menor a ingestão dos nutrientes maior é o risco de desenvolvimento de sarcopenia.

Palavras-chave: Envelhecimento, composição corporal, força muscular, consumo alimentar, doença renal crônica.

\section{ASSOCIATION BETWEEN FOOD INTAKE AND RISK OF SARCOPENIA IN ELDERLY PATIENTS ON HEMODIALYSIS.}

\begin{abstract}
The association between food intake and risk of sarcopenia in elderly patients with chronic kidney disease (CKD) on hemodialysis was verified. Elderly patients of both sexes were recruited from the hemodialysis sector of two hospitals in the interior of São Paulo. To compose the group, all individuals should have a diagnosis of CKD and undergo hemodialysis. Participants $(n=25)$ had their anthropometric measurements evaluated, followed by the application of the SARC-F questionnaire + calf circumference (CC) to identify the risk of sarcopenia. In addition, patients responded to a food diary to assess food intake. Associations were found between daily intake of calories, carbohydrates, phosphorus, iron, sodium and SARC-F + CC values. It was concluded that there was an association between some factors of food intake and SARC-F + CC, so that the lower the intake of nutrients, the greater the risk of developing sarcopenia.
\end{abstract}

Keywords: Aging, body composition, muscle strength, food consumption, chronic kidney disease.

\section{INTRODUÇÃO}

A doença renal crônica (DRC) consiste na perda progressiva, lenta e irreversível da estrutura e função dos rins, levando a incapacidade do organismo de manter o equilíbrio eletrolítico ${ }^{1}$. 0 risco de desenvolvimento da DRC está diretamente Colloq Vitae 2020 set-dez; 12(3): 16-25.

Artigo Open Access sob uma licença CC BY-NC-ND (http://creativecommons.org/licenses/by-nc-nd/4.0/). associado com as doenças cardiovasculares, como a hipertensão arterial e a diabetes mellitus, e estudos mostram que o envelhecimento e o histórico familiar da doença também se fazem presentes neste contexto, sendo que indivíduos com idade acima de 60 anos com proteinúria, 
dislipidemia e tabagismo podem apresentar uma progressão acelerada da doença $a^{1-3}$.

A literatura aponta que 0 melhor indicador para determinar a DRC é a taxa de filtração glomerular (TFG) ${ }^{4}$. Contudo, para melhor entendimento e diagnóstico dos riscos e complicações voltados ao comprometimento renal, a diretriz para DRC recomenda a avaliação da albuminúria. Dessa forma, os critérios utilizados são albuminúria $>30 \mathrm{mg} / 24 \mathrm{~h}$ ou relação albumina/creatinina $<30 \mathrm{mg} / \mathrm{g}$ e TFG $<60$ $\mathrm{ml} / \mathrm{min} / 1,73 \mathrm{~m}^{2}$, além dos demais critérios por um período maior que 3 meses ${ }^{5}$.

Com o avanço da doença, o indivíduo pode chegar a fase terminal de insuficiência renal crônica (TFG inferior a $15 \mathrm{ml} / \mathrm{min} / 1,73 \mathrm{~m}^{2}$ ) que corresponde ao estágio mais crítico e avançado de perda funcional do rim $^{6}$. Nessa fase, há a necessidade de realização de tratamento dialíticos, sendo diálise peritoneal (DP) ou hemodiálise, os quais desempenham o papel dos rins como excreção dos metabólitos uréia, creatina e ácido úrico ${ }^{7}$.

Um ponto de suma importância relacionado a DRC é o estado nutricional debilitado apresentado pelos pacientes, capaz de levar a um aumento do risco de mortalidade, principalmente aqueles que se encontram em tratamento de hemodiálise ${ }^{8}$. Além disso, esse estado nutricional favorece um quadro de pior evolução clínica, dificulta a reabilitação, promove maior quadro de infecções e compromete a qualidade de vida desse indivíduos ${ }^{9}$.

Os mecanismos envolvidos no processo de desnutrição nos pacientes com DRC não estão completamente elucidados, entretanto acreditase que o estado inflamatório crônico, a restrição dietética e os níveis de sedentarismo, têm papel importante na fisiopatologia deste estado nutricional ${ }^{10}$. Esses achados, associados com o envelhecimento, se mostram como um dos fatores que levam ao quadro de perda de massa muscular e tornam esses indivíduos propensos a desenvolver sarcopenia ${ }^{11}$, que é caraterizada pela perda de massa e força/função muscular começando a partir dos 40 anos com queda de $0,5 \%$ ao ano e aumentando até cerca de $1 \%$ ao ano a partir dos 65 anos de idade ${ }^{12}$.

Essa perda de massa muscular está associada com diminuição da qualidade de vida ${ }^{13}$ e da capacidade funcional ${ }^{14}$ em pacientes em hemodiálise, que por sua vez, leva a perpetuação do estilo de vida sedentário, favorecendo um círculo vicioso de perda de massa muscular, Colloq Vitae 2020 set-dez; 12(3): 16-25.

Artigo Open Access sob uma licença CC BY-NC-ND (http://creativecommons.org/licenses/by-nc-nd/4.0/). aumentando as chances de complicações cardiovasculares e a taxa de mortalidade ${ }^{15}$. Além disso, estudos vêm demonstrando que, quanto maior o tempo, em anos, de diálise, maior é a probabilidade de os pacientes virem a desenvolver desnutrição e sarcopenia ${ }^{16,17}$.

Levando em consideração os danos causados pela hemodiálise e consequentemente o aumento do risco de morbimortalidade desses pacientes, faz necessário a avaliação nutricional, a qual se mostra essencial no se diz respeito a prevenção da desnutrição ${ }^{18}$, bem como avaliação do risco para desenvolvimento de sarcopenia, a fim de elaborar estratégias para melhorar o estado nutricional, que caracteriza-se como um dos principais objetivos do tratamento em pacientes em hemodiálise ${ }^{19}$, o que justifica a realização do presente estudo.

Ademais, destaca-se como importante o fato de que a sarcopenia apresenta-se como um crescente problema de saúde pública ${ }^{20}$, que como consequência eleva os custos para o sistema de saúde. Diante disso, o objetivo do presente estudo foi verificar a associação entre ingestão alimentar e risco de sarcopenia em pacientes idosos com DRC em hemodiálise.

\section{MÉTODOS \\ NATUREZA DA PESQUISA}

Foi realizado um estudo descritivo de caráter transversal, desenvolvido com os pacientes idosos portadores de DRC. O projeto foi devidamente cadastrado na Plataforma Brasil, aprovado pelo Comitê de Ética em Pesquisa (CEP) da Unoeste ( $n$ - CAEE: 21856819.3.0000.5515) e seguiu as orientações do Conselho Nacional de Ética em Pesquisa (CONEP) baseadas na Declaração de Helsinque. Para realização do estudo, foi elaborado um Termo de Consentimento Livre e Esclarecido (TCLE), de acordo com modelo preconizado pela CEP/UNOESTE, o qual foi assinado pelos participantes da pesquisa.

Participantes

Para compor o grupo, todos os indivíduos deveriam possuir diagnóstico de DRC em seu prontuário, realizar hemodiálise e atender aos seguintes critérios de inclusão: 1) possuir idade maior ou igual a 60 anos, 2) não ser portador de neoplasias, insuficiência hepática, infecção aguda ou crônica ou demais patologias que pudessem alterar de maneira significante o estado nutricional do paciente. Os pacientes que não 
aceitaram assinar o TCLE, ou não responderam o diário alimentar, não fizeram parte da amostra.

Procedimento experimental

Inicialmente as pesquisadoras fizeram um levantamento dos pacientes que possuíam idade acima ou iguais a 60 anos dos serviços de hemodiálise de dois hospitais do interior paulista. Em seguida, esses pacientes foram abordados e as pesquisadoras explicaram sobre os procedimentos do estudo e convidaram para participação do mesmo.

Os pacientes que aceitaram participar da pesquisa assinaram o TCLE. Logo após tiveram suas medidas antropométricas avaliadas, seguida da aplicação do questionário para identificação do risco de sarcopenia. Ainda, os pacientes receberam um diário alimentar para avaliação da ingestão alimentar, o qual foi realizado em casa pelo próprio paciente ou familiares no caso de analfabetismo, e entregue na semana seguinte para as pesquisadoras.

Avaliação antropométrica

A avaliação antropométrica foi realizada 30 minutos após a sessão de hemodiálise ${ }^{21}$. Os voluntários verificaram o peso corporal por meio de uma balança digital (Plenna, TIN 00139 MAXIMA, Brasil) e altura, por meio de um estadiômetro (ES 2020 - Sanny, Brasil) ${ }^{21}$ e tiveram seu índice de massa corporal (IMC) calculado dividindo o peso corporal pelo quadrado da estatura. Foram considerados os valores de IMC segundo os pontos de corte de Lipschitz ${ }^{22}$ onde os indivíduos foram classificados como baixopeso quando apresentaram $\mathrm{IMC}<22 \mathrm{~kg} / \mathrm{m}^{2}$, eutrofia quando IMC entre $22 \mathrm{~kg} / \mathrm{m}^{2}$ e $27 \mathrm{~kg} / \mathrm{m}^{2}$ e sobrepeso quando IMC $>27 \mathrm{~kg} / \mathrm{m}^{2}$.

Em seguida os pacientes tiveram a circunferência da panturrilha (CP), circunferência do braço (CB) e dobra cutânea tricipital (DCT) verificados. Essas medidas foram descritas a seguir.

A CP foi verificada na área de maior circunferência da panturrilha esquerda, estando o peso corporal igualmente distribuído nos membros inferiores. Os pontos de corte para detecção de massa muscular diminuída em idosos foi de $34 \mathrm{~cm}$ para homens e $33 \mathrm{~cm}$ para mulheres ${ }^{23}$.

Para CB, utilizando uma trena antropométrica, foi medida no braço esquerdo a distância entre a projeção lateral do processo acromial da escápula e a margem inferior do processo olecraniano da ulna, com cúbito flexionado a 90 ․ Em seguida o ponto médio Colloq Vitae 2020 set-dez; 12(3): 16-25.

Artigo Open Access sob uma licença CC BY-NC-ND (http://creativecommons.org/licenses/by-nc-nd/4.0/). dessa medida foi marcado e com os braços soltos para os lados e a palma das mãos viradas para as coxas, a fita foi aplicada firmemente ao redor do braço $^{23}$. Para avaliação da massa muscular, a circunferência muscular do braço (CMB) foi calculada por meio da fórmula $\mathrm{CMB}=\mathrm{CB}-(0,314$ $x$ DCT) e sua adequação foi realizada por meio da fórmula $\% \mathrm{CMB}=\mathrm{CMB} / \mathrm{CMB} 50 \times 100$, onde $\mathrm{O}$ valor de referência foi obtido no percentil 50을 definido para a população geral de acordo com Frisancho ${ }^{25}$. De acordo com o resultado da CMB, o paciente foi classificado da seguinte forma: desnutrição Grave, para valores < 70\%; desnutrição Moderada, para valores de $70-80 \%$; desnutrição Leve, para valores de $80-90 \%$; adequado, para valores de $90-110 \%$; excesso de peso, para valores de $>110 \%{ }^{26}$.

Por fim, para DCT, a prega cutânea foi destacada $1 \mathrm{~cm}$ acima do nível marcado para a mensuração da $\mathrm{CB}$. Foram realizadas três medicas consecutivas e a média aritmética considerada como resultado final, expressa em milímetros por meio de um adipômetro (Sanny American Medical do Brasil) $)^{24}$. A adequação do percentual de DCT foi obtida por meio da fórmula: \%DCT = DCT/DCT 50 x 100 e o valor de referência obtido do percentil 50으 para população em geral de acordo com Frinsancho ${ }^{25}$ e classificado como desnutrição grave, para valores < 70\%; desnutrição moderada, para valores de $70-80 \%$; desnutrição leve, para valores de $80-90 \%$; adequado, para valores de $90-110 \%$ e excesso de peso, para valores de $110-120 \%$; obesidade, para valores $>120 \%{ }^{27}$.

Questionário SARC-F + CP

Para verificação do risco de sarcopenia foi utilizado o questionário SARC-F + CP proposto por Barbosa-Silva et al. ${ }^{28}$. Este questionário apresenta cinco perguntas objetivas sobre força, deambulação, ato de levantar-se de uma cadeira, subir um lance de escadas e quedas e possui a verificação da $\mathrm{CP}$, podendo possuir valor de 0 a 20 pontos. Como resultado, pode-se classificar o paciente em dois escores: 1) 0 a 10 pontos - sem sinais sugestivos de sarcopenia no momento e 2) 11 a 20 pontos - sugestivo de sarcopenia.

Diário alimentar

Para a investigação do consumo alimentar foi realizado o diário alimentar, preenchido pelo paciente em 3 dias não consecutivos, sendo considerado 1 dia de final de semana. O diário alimentar é considerado uma intervenção de curta duração e fácil logística, que mede o consumo alimentar atual do paciente. 
Além disso, possui como vantagens o fato de não depender da memória do paciente, apresentando menor erro quando há orientação detalhada para - registro ${ }^{29}$. Para avaliação do consumo alimentar, os dados foram tabulados no programa DietBox ${ }^{\circledR}$.

Análise estatística

A caracterização da amostra foi realizada por meio de estatística descritiva e os resultados foram apresentados em valores de média e desvio padrão. A normalidade dos dados foi verificada por meio do teste de Shapiro-wilk. Para comparação dos dados entre os pacientes com risco para sarcopenia e sem risco para sarcopenia foi utilizado teste T-Student e Mann-Whitney, e para associação entre os valores encontrados no SARC-F + CP e os demais dados de ingestão alimentar e variáveis antropométricas foram utilizados o teste de correlação de Pearson para dados paramétricos e de Spearman para dados não paramétricos. Todas as análises estatísticas foram realizadas utilizando o software Statistical Package for the Social Sciences (SPSS) versão 16.0 e a significância estatística adotada foi de $5 \%$.

\section{RESULTADOS}

A caracterização da amostra total de pacientes com DRC em hemodiálise, por meio de valores médios com seus respectivos desvios padrões para idade, tempo de hemodiálise e variáveis antropométricas, pode ser observada na tabela 1. Foram avaliados 25 pacientes, sendo 21 homens e 4 mulheres.

Tabela 1. Caracterização da amostra total de pacientes com DRC em hemodiálise.

\begin{tabular}{ll}
\hline Variável $(\mathbf{n}=\mathbf{2 5})$ & Média \pm desvio padrão \\
\hline Idade (anos) & $72 \pm 6,51$ \\
Tempo de Hemodiálise (meses) & $28,17 \pm 27,19$ \\
Peso $(\mathrm{Kg})$ & $74,77 \pm 15,15$ \\
Altura $(\mathrm{m})$ & $1,65 \pm 0,06$ \\
$\mathrm{IMC}\left(\mathrm{kg} / \mathrm{m}^{2}\right)$ & $27,34 \pm 4,88$ \\
Adequação CB (\%) & $107,17 \pm 19,55$ \\
Adequação de CMB (\%) & $94,53 \pm 17,96$ \\
Adequação de DCT $(\%)$ & $224,07 \pm 81,09$ \\
CP $(\mathrm{cm})$ & $33,91 \pm 3,75$ \\
\hline
\end{tabular}

Legenda: $\mathrm{IMC}$ = índice de massa corpórea; $\mathrm{CB}=$ circunferência do braço; $\mathrm{CMB}=$ circunferência muscular do braço; $\mathrm{DCT}=$ dobra cutânea tricipital; $\mathrm{CP}=$ circunferência da panturrilha; \% = porcentagem; $\mathrm{cm}=$ centímetros; $\mathrm{m}=$ metros; $\mathrm{kg} / \mathrm{m}^{2}=$ quilos por metro ao quadrado; $\mathrm{kg}$ = quilos.

Pode ser verificado na tabela 2, que houveram associações negativas entre valores de SARC-F +CP e ingestão de calorias/dia, carboidrato, fósforo, ferro e sódio. Porém, sem associações para as demais variáveis de ingestão alimentar.

Tabela 2. Correlação entre risco para sarcopenia, tempo de hemodiálise e ingestão alimentar $(n=25)$.

\begin{tabular}{lll}
\hline & \multicolumn{2}{l}{ Correlação (variável dependente: SARC-f + CP) } \\
\cline { 2 - 3 } Variáveis independentes & $\mathbf{R}$ & $\boldsymbol{p}$-valor \\
\hline Tempo de hemodiálise & 0,296 & 0,161 \\
Calorias/dia & $-0,471$ & $0,020^{*}$ \\
Proteína (g) & $-0,382$ & 0,066 \\
Carboidrato (g) & $-0,438$ & $0,032^{*}$ \\
Lipídio (g) & $-0,295$ & 0,162 \\
Cálcio (mg) & $-0,072$ & 0,740 \\
Fosforo (mg) & $-0,424$ & $0,039^{*}$ \\
Ferro (mg) & $-0,462$ & $0,023^{*}$ \\
Sódio (mg) & $-0,557$ & $0,005^{*}$ \\
Potássio (mg) & $-0,251$ & 0,237
\end{tabular}

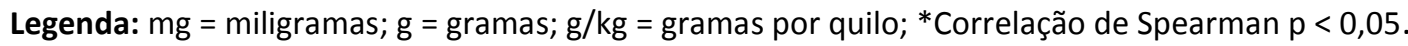


Na tabela 3, observa-se a comparação entre os pacientes quando divididos em dois grupos: grupo sugestivo para sarcopenia e não sugestivo para sarcopenia de acordo com os valores encontrados no SARC-f $+\mathrm{CP}$.
Comparações entre idade, tempo de hemodiálise, variáveis antropométricas e variáveis de ingestão alimentar foram realizadas para os grupos.

Tabela 3. Comparação das variáveis dos grupos Sugestivo para sarcopenia e não sugestivo para sarcopenia.

\begin{tabular}{|c|c|c|c|c|c|}
\hline \multirow[t]{2}{*}{ Variável } & \multicolumn{2}{|c|}{$\begin{array}{l}\text { Sugestivo para sarcopenia } \\
(n=8)\end{array}$} & \multicolumn{2}{|c|}{$\begin{array}{l}\text { Não } \quad \text { sugestivo } \\
\text { sarcopenia }(n=16)\end{array}$} & \multirow[t]{2}{*}{$\begin{array}{l}\text { para } \\
p \text {-valor }\end{array}$} \\
\hline & Média & $\pm \mathrm{DP}$ & Média & $\pm \mathrm{DP}$ & \\
\hline Idade (anos) & 75,25 & 6,92 & 70,38 & 5,84 & 0,083 \\
\hline $\begin{array}{l}\text { Tempo de Hemodiálise } \\
\text { (meses) }\end{array}$ & 40,38 & 35,12 & 22,06 & 20,96 & 0,062 \\
\hline Peso (Kg) & 67,84 & 12,53 & 78,24 & 15,50 & 0,115 \\
\hline Altura (m) & 1,66 & 0,05 & 1,65 & 0,07 & 0,853 \\
\hline $\mathrm{IMC}\left(\mathrm{kg} / \mathrm{m}^{2}\right)$ & 24,79 & 4,61 & 28,62 & 4,62 & 0,068 \\
\hline Adequação CB (\%) & 105,92 & 19,83 & 107,80 & 20,03 & 0,830 \\
\hline Adequação de CMB (\%) & 93,31 & 18,35 & 95,14 & 18,34 & 0,820 \\
\hline Adequação de DCT (\%) & 225,66 & 77,07 & 223,27 & 85,49 & 0,830 \\
\hline $\mathrm{CP}(\mathrm{cm})$ & 30,44 & 2,13 & 35,64 & 3,12 & $0,000^{*}$ \\
\hline SARC-F + CP & 13,50 & 1,77 & 4,63 & 4,32 & $0,000 * *$ \\
\hline Calorias/dia & 1979,46 & 408,30 & 2384,15 & 630,48 & 0,115 \\
\hline Proteína (g) & 84,84 & 19,16 & 112,08 & 44,97 & 0,142 \\
\hline Carboidrato (g) & 214,79 & 63,35 & 272,42 & 59,83 & $0,040^{* *}$ \\
\hline Lipídio (g) & 86,41 & 42,37 & 92,22 & 39,47 & 0,759 \\
\hline Cálcio (mg) & 440,69 & 189,29 & 509,79 & 169,30 & 0,374 \\
\hline Fosforo (mg) & 919,50 & 217,90 & 1176,27 & 367,95 & 0,084 \\
\hline Ferro (mg) & 15,83 & 3,96 & 19,48 & 4,16 & 0,062 \\
\hline Adequação de DCT (\%) & 225,66 & 77,07 & 223,27 & 85,49 & 0,830 \\
\hline Sódio (mg) & 2176,23 & 323,13 & 3154,95 & 883,70 & $0,007^{* *}$ \\
\hline Potássio (mg) & 1886,27 & 388,54 & 2382,83 & 779,70 & 0,106 \\
\hline Proteína (g/kg) & 1,26 & 0,29 & 1,49 & 0,65 & 0,358 \\
\hline
\end{tabular}

Legenda: $\mathrm{IMC}=$ índice de massa corpórea; $\mathrm{CB}=$ circunferência do braço; $\mathrm{CMB}=$ circunferência muscular do braço; $\mathrm{DCT}=$ dobra cutânea tricipital; $\mathrm{CP}=$ circunferência da panturrilha; $\mathrm{cm}=$ centímetros; $\mathrm{m}=$ metros; $\mathrm{kg} / \mathrm{m}^{2}=$ quilos por metro ao quadrado; $\mathrm{kg}=$ quilos; $\mathrm{mg}=$ miligramas; $\mathrm{g}=$ gramas; $\mathrm{g} / \mathrm{kg}=$ gramas por quilo; $\mathrm{SARCF}=$ Questionário de sarcopenia. * Teste T-Student; ${ }^{* *}$ Teste de Mann - Whitney; $\mathrm{P}<0,05$.

\section{DISCUSSÃO}

O objetivo do presente estudo foi verificar a associação entre ingestão alimentar e risco para sarcopenia em pacientes idosos com DRC em hemodiálise. Houve associação entre risco para sarcopenia e os seguintes fatores relacionados a ingestão alimentar: calorias/dia, total em gramas de carboidrato, fósforo, ferro e sódio. $E$ essas associações foram negativas, de forma que quanto menor o valor desses fatores maior o valor de SARC-F +CP, ou seja, maior risco para sarcopenia.

Nesse estudo, os idosos em hemodiálise apresentaram média de idade acima de 70 anos. No entanto, em valores médios, o grupo sugestivo para sarcopenia apresentou maior idade, o que era esperado uma vez que essa condição parece ocorrer com mais frequência conforme a idade aumenta. Mendes et al. ${ }^{30}$, avaliaram 53 idosos sedentários e encontraram que aqueles que eram sarcopênicos 
apresentavam também maior idade, com uma média de 71 anos em comparação com idosos não sarcopênicos que apresentavam em média 66 anos.

Sabe-se que a hemodiálise é um processo que pode levar a alterações no estado nutricional dos indivíduos devido a inflamação, perda de nutrientes para o dialisato, como aminoácidos ${ }^{31}$, sedentarismo e por fim ainda pode ocorrer situações como acidose metabólica que induz catabolismo no paciente, portanto, quanto maior o tempo de hemodiálise possivelmente também maiores são as alterações ${ }^{32}$. Apesar disso, não foi encontrada associação com os valores de SACR- $f$ $+\mathrm{CP}$.

Apesar de maior tempo de hemodiálise e as possíveis consequências nos pacientes sugestivos para sarcopenia, esses indivíduos apresentaram-se eutróficos pelo IMC. Ao contrário de nosso achado, Torres et al. ${ }^{32}$, mostraram que a maioria dos idosos em hemodiálise apresentaram magreza. No entanto, o IMC não deve ser considerado isoladamente por não expressar a distribuição dos tecidos corporais. Nesse sentido, devem ser observados também os demais dados antropométricos ${ }^{34}$. Para circunferência de panturrilha, que é um marcador de reserva muscular, foi encontrado valor menos que $33 / 34 \mathrm{~cm}$ que são os valores utilizados de maneira isolada. Sabe-se que CP inferior a $31 \mathrm{~cm}$ é atualmente considerado o melhor indicador clínico de sarcopenia, estando associado a incapacidade funcional e risco de quedas $^{35}$.

Em relação a $\mathrm{CB}$, que reflete estado geral, e CMB que reflete os compartimentos de reserva proteico do organismo, os valores percentuais dessas medidas indicaram eutrofia. E DCT que reflete a reserva de gordura corporal total indicou obesidade. No entanto, no estudo de Torres et al. ${ }^{33}$, com uma população de adultos e idosos, a desnutrição leve foi o que mais prevaleceu entre os resultados obtidos na $C B$ e CMB e a desnutrição grave para DCT, sendo justificados pela inadequada ingestão calórica e proteica, que acarreta em carência nutricional e consequentemente perda de massa muscular. Os resultados encontrados no presente estudo podem ser justificados, em parte, pelo excesso de pele encontrado na região do braço dos indivíduos, característica que é comum nessa faixa etária e também por edema que pode ter permanecido nos pacientes após a hemodiálise, interferindo no resultado real dessas medidas.
A respeito da ingestão alimentar, houve associação negativa entre calorias/dia, quantidade de carboidrato e os valores de SARC- $F$ $+\mathrm{CP}$, mostrando que quanto menor a ingestão de calorias e carboidratos, maior eram os valores encontrados no questionário de sarcopenia. Os indivíduos do grupo sugestivo para sarcopenia ingeriram em média menos calorias/dia e menores quantidades de macronutrientes do que o grupo não sugestivo para sarcopenia, com diferença estatística somente para carboidrato. Para ambos os grupos, os valores de carboidratos estão abaixo do recomendado, sendo que para essa população o ideal seria um consumo de cerca de 30 a $35 \mathrm{kcal} / \mathrm{kg}$ e $50-60 \%$ de carboidratos $^{36}$. Já para os lipídios recomenda-se cerca de $25-30 \%$, apresentando ingestão elevada no presente estudo de acordo com o valor energético total ${ }^{37}$.

Esses achados podem estar relacionados às dietas ingeridas por esses pacientes, que devido às adequações, podem ser insípidas e monótonas. Além disso, existe uma relação entre síndrome urêmica e ingestão alimentar reduzida devido a distúrbios gastrointestinais como anorexia, náuseas, vômito, e hálito urêmico $^{38}$, no entanto no presente estudo não foram avaliados aspectos relacionados com a síndrome urêmica. Nesse sentindo, acredita-se que a menor ingesta de calorias e macronutrientes podem levar a menor massa magra nessa população, auxiliando no desenvolvimento de sarcopenia ${ }^{33}$.

Analisando a proteína, os pacientes do grupo sugestivo para sarcopenia ingeriram em média valores que estão dentro das recomendações, porém não foi o suficiente para manutenção da massa magra, o que foi observado pelos valores de CP. Isso pode ser justificado considerando que no tratamento de hemodiálise ocorrem perdas de 2 a $3 g$ de proteína e 4 a $8 \mathrm{~g}$ de aminoácidos. Além disso, os níveis séricos de albumina diminuem em situações de hipervolemia, o que é muito frequente em pacientes em tratamento dialítico ${ }^{39}$. Um estudo avaliando pacientes em hemodiálise encontrou ingestão proteica de $1,1 \mathrm{~g} / \mathrm{Kg}$, sendo considerado um valor insuficiente para essa população, pois pode levar a um balanço nitrogenado negativo, gerando, portanto, perda de massa muscular ${ }^{40}$.

Além disso, é importante destacar que a maioria dos pacientes em hemodiálise são sedentários, o que pode acabar contribuindo ainda mais para a perda de massa magra. Em 
uma recente revisão, os autores encontraram que o exercício resistido melhorou o ganho de força nos indivíduos de idade mais avançada que apresentavam sarcopenia, consequentemente melhorando marcha e equilíbrio, sendo considerado também um estímulo importante para a ganho de massa muscular ${ }^{41}$.

Sobre os micronutrientes, foram encontrados valores inadequados de ingestão de cálcio segundo o parâmetro nutricional recomendado que é em torno de $1.000 \mathrm{mg}$ por $\mathrm{dia}^{42}$. LUZ et al. $^{43}$ encontraram resultados semelhantes ao presente estudo com pacientes em hemodiálise. 0 consumo desse micronutriente pode requerer suplementação devido a sua absorção intestinal diminuída e em virtude do baixo consumo de alimentos fontes como leites e derivados, uma vez que esses em geral também são fontes de fósforo ${ }^{42}$.

No presente estudo, a ingestão de fósforo estava adequada, de acordo com as recomendações da Sociedade Brasileira de Nefrologia ${ }^{44}$ que indica valores de 800 a 1200 mg diárias. Em quantidades elevadas esse nutriente associa-se ao desenvolvimento de hipertireoidismo secundário e calcificação de tecidos moles nesses pacientes, porém como as recomendações de proteínas são elevadas, há necessidade na grande parte das vezes do uso de quelantes $^{42}$.

A respeito do ferro, sabe-se que sua deficiência é comum na DRC, devido a diminuição de eritropoietina nessa população, bem como pela menor absorção intestinal ${ }^{45}$ e também pela perda no processo de hemodiálise, sendo a causa mais comum de anemia. No presente estudo o consumo de ferro foi relativamente alto em comparação com a recomendação diária para dialíticos, que varia em torno de $8 \mathrm{mg}$ para homens e $15 \mathrm{mg}$ para mulheres ${ }^{46}$. No entanto, não observamos análises bioquímicas para verificação da presença de anemia, o que poderia fornecer informações adicionais sobre possíveis relações com a perda de massa magra.

Em relação ao consumo de sódio, encontrou-se valor elevado segundo as recomendações diárias de até $2.300 \mathrm{mg} / \mathrm{dia}$, ou $6 \mathrm{~g}$ de sal/dia ${ }^{47}$. Essa maior ingestão de sódio pode estar relacionada ao aumento da pressão arterial, presença de edemas e aumento de sede nesses pacientes. Uma maior ingestão de sódio associada com aumento de ingestão de líquidos pode levar a um aumento do IMC, associado ao ganho de peso interdialítico excessivo, o que ocorreu em um estudo de Koehnlein et al. ${ }^{48}$.

A presença da associação negativa de fósforo, ferro e sódio com os valores de SARC-f + $\mathrm{CP}$ parece estar mais relacionada as menores quantidades ingeridas dos macronutrientes do que necessariamente a seus valores reais com a presença de sarcopenia.

Por fim, a respeito do potássio, é de suma importância que sua ingestão seja controlada, pois a hipercalemia pode resultar em arritmia, parada cardíaca, ou, ainda, levar à paralisia dos músculos que controlam a ventilação pulmonar. Luz et al. ${ }^{43}$, assim como nesse estudo, verificaram valores médios de consumo de potássio abaixo do recomendado para pacientes em hemodiálise que é de 1950 a $2730 \mathrm{mg}^{47}$.

A importância desse estudo foi fundamental por demostrar que o consumo alimentar dos pacientes em hemodiálise pode contribuir para alteração do seu estado nutricional, apresentando muitas vezes perda de massa magra e consequentemente perda de força muscular, levando a situações como sarcopenia, a qual gera riscos como quedas, e ainda piora a qualidade de vida dos pacientes. Esse estudo também apresenta limitações, pois análises bioquímicas poderiam auxiliar na interpretação dos dados e sabe-se que a avaliação antropométrica nesses pacientes é limitada devido ao edema que ocorre pela perda da função renal.

A partir dos resultados obtidos, conclui-se que houve associação, mesmo que fraca, entre ingestão de calorias/dia, carboidratos, fósforo, ferro e sódio e valores de SARC-F + CP, de maneira que quanto menor a ingestão desses nutrientes maior é o valor encontrado no questionário, ou seja, maior o risco de desenvolvimento de sarcopenia em pacientes idosos em hemodiálise.

\section{AGRADECIMENTOS}

Agradecemos a todos os participantes da pesquisa.

\section{CONFLITO DE INTERESSE}

Os autores declaram não haver qualquer potencial conflito de interesse que possa interferir na imparcialidade deste trabalho científico. 


\section{REFERÊNCIAS}

1. Romão Junior JE. Doença Renal Crônica: Definição, Epidemiologia e Classificação. Braz J Nephrol 2004; 26(3 suppl. 1):1-3.

2. Webster AC, Nagler EV, Morton RL, Masson P. Chronic Kidney Disease. Lancet. 2017;;389(10075):1238-52. https://doi.org/10.1016/S0140-6736(16)32064-5

3. Sarnak MJ. Cardiovascular complications in chronic kidney disease. Am J Kidney Dis. 2003; 41(5 Suppl):11-7. https://doi.org/10.1016/s02726386(03)00372-x

4. Levey AS, Becker C, Inker LA. Glomerular filtration rate and albuminuria for detection and staging of acute and chronic kidney disease in adults: a systematic review. JAMA. 2015; 313:837-46.

https://doi.org/10.1001/iama.2015.0602

5. Kirsztajn GM, Salgado FN, Draibe SA, Netto MVP, Thome FS, Souza E, et al. Fast Reading of the KDIGO 2012: Guidelines for evaluation and management of chronic kidney disease in clinical practice. J Bras Nefrol. 2014; 36(1):63-73. http://doi.org/10.5935/0101-2800.20140012

6. João ERJ, Riella MC, Martins C, Baxmann AC, Vavruk AM, Piccolo AP. Nutrição e o Rim. 2a. ed. Rio de Janeiro: Guanabara Koogan; 2013.

7. Machado GRG, Pinhati FR. Tratamento de diálise em pacientes com insuficiência renal crônica. Cadernos Unifoa. 2014; 9(26).

8. Afsar B, Sezer S, Arat Z, Tutal E, Ozdemir FN, Haberal M. Reliability of mini nutritional assessment in hemodialysis compared with subjective global assessment. J Renal Nutr. 2006; 16:277-82.

https://doi.org/10.1053/i.jrn.2006.01.012

9. Pupim LB, Ikizler TA. Assessment and monitoring of uremic malnutrition. J Renal Nutr 2004;

14:6-19.

https://doi.org/10.1053/j.jrn.2003.10.001.

10.O'Hare AM, Tawney K, Bacchetti P, Johansen $\mathrm{KL}$. Decreased survival among sedentary patients undergoing dialysis: results from the dialysis morbidity and mortality study wave 2. Am J
Kidney Dis. 2003; 41(2):447-54. https://doi.org/10.1053/ajkd.2003.50055

11.Greco A, Paroni G, Seripa D, Addante F, Dagostino MP, Aucella F. Frailty, disability and physical exercise in the aging process and in chronic kidney disease. Kidney Blood Pressure Res. 2014; 39:164-8.

https://doi.org/10.1159/000355792

12.Cruz-Jentoft AJ, Baeyens JP, Bauer JM, Boirie $Y$, Cederholm T, Landi F, et al. Sarcopenia: European consensus on definition and diagnosis: Report of the European Working Group on Sarcopenia in older people. Age Ageing. 2010; 39:412-23. https://doi.org/10.1093/ageing/afq03

13. Noori N, Kopple JD, Kovesdy CP, Feroze U, Sim JJ, Murali SB, et al. Mid-arm muscle circumference and quality of life and survival in maintenance hemodialysis patients. Clin J Am Soc Nephrol. 2010; 5(12):2258-68. https://doi.org/10.2215/CJN.02080310

14.Fouque D, Kalantar-Zadeh K, Kopple J, Cano N, Chauveau P, Cuppari L, et al. A proposed nomenclature and diagnostic criteria for proteinenergy wasting in acute and chronic kidney disease. Kidney Int. 2008; 73(4):391-8. https://doi.org/10.1038/sj.ki.5002585

15.Fahal IH. Uraemic sarcopenia: etiology and implications. Nephrol Dialysis Transplant. 2014; 29:1655-65. https://doi.org/10.1093/ndt/gft070

16.Chazot C, Laurent G, Charra B, Blanc C, VoVan $C$, Jean $G$, et al. Malnutrition in long-term haemodialysis survivors. Nephrol Dial Transplant. 2001; 16(1):61-9. https://doi.org/10.1093/ndt/16.1.61

17.Greco A, Paroni G, Seripa D, Addante F, Dagostino MP, Aucella F. Frailty, disability and physical exercise in the aging process and in chronic kidney disease. Kidney Blood Pressure Res. 2014; 39:164-8. https://doi.org/10.1159/000355792

18. Martins C, Riella MC. Nutrição em hemodiálise. In: Riella MC, Martins C. Nutrição e o rim. Rio de Janeiro: Guanabara Koogan; 2001. pp.114-31.

19.Bayo MP, Granados JJQ, Ortega AO, Peinado CA, Lópes MJO, Serrana HLG, et al.. Estudio 
longitudinal del índice de massa corporal (IMC) em pacientes em diálisis. Nutr Hosp 2006; 21:155-62.

20.Veras RP. Estratégias para o enfrentamento das doenças crônicas: um modelo em que todos ganham. Rev Bras Geriatr Gerontol. 2011; 14(4): 779-86. $\quad$ https://doi.org/10.1590/S1809$\underline{98232011000400017}$

21.Rodrigues J, Santin F, Barbosa Brito FS, Carrero JJ, Lindholm B, Cuppari L, et al. Sensitivity and specificity of body mass index as a marker of obesity in elderly patients on hemodialysis. J Ren Nutr. 2016; 26:65-71. https://doi.org/10.1053/i.jrn.2015.09.001

22.Lipschitz DA. Screening for nutritional status in the elderly. Prim Care. 1994; 21:55-67.

23.Pagotto V, Santos KF, Malaquias SG, Bachion MM, Silveira EA. Calf circumference: clinical validation for evaluation of muscle mass in the elderly. Rev Bras Enferm 2018; 71(2):322-8. https://doi.org/10.1590/0034-7167-2017-0121

24. Heyward VH, Stolarczyk LM. Método Antropométrico. In: Heyward VH, Stolarczyk, editors. Avaliação da composição corporal aplicada. São Paulo: Manole; 2000. p.73-95.

25. Frisancho AR. New norms of upper limb fat and muscle areas for assessment of nutritional status. Am J Clin Nutr. 1981; 34(11):2540e5. https://doi.org/10.1093/ajcn/34.11.2540

26.Blackburn GL, Thornton PA. Nutritional assessment of the hospitalized patient. Med Clin North Am. 1979; 63:11103-15. https://doi.org/10.1016/S0025-7125(16)31663-7

27. Martins C, Riella MC. Nutrição e hemodiálise. In: Riella MC, Martins CM. Nutrição e o rim. Rio de Janeiro: Guanabara Koogan; 2011. p.114-31.

28.Barbosa-Silva G, Menezes R.M, et al. Enhancing SARC-F: Improving sarcopenia screening in the clinical practice. J Am Med Dir Assoc. 2016; 17(12):1136-41. https://doi.org/10.1016/i.jamda.2016.08.004

29. Fisberg RM, Martini LA, Slater B. Métodos de inquéritos alimentares: métodos e bases científicos. São Paulo: Manole; 2005. p. 1-31.
30.Mendesa GS, Teixeira THMM, Souza VC, Neiva TS, Pereira KP, Landima MFT et al. Sarcopenia em idosos sedentários e sua relação com funcionalidade e marcadores inflamatórios. Geriatr Gerontol Aging. 2016; 10(1):23-8. https://doi.org/10.5327/Z2447$\underline{2115201600010005}$

31. Kamimura MA, Draibe SA, Sigulem DM, Cuppari L. Métodos de avaliação da composição corporal em pacientes submetidos à hemodiálise. Rev Nutr. 2004; 17(1):97-105. https://doi.org/10.1590/S1415$\underline{52732004000100011}$

32. Bonfim RF. Prevalência de sedentarismo e fadiga entre os portadores de doença renal crônica em hemodiálise e efeito do exercício intradialítico sobre a eficácia da hemodiálise. 2009. 117 f. [dissertação]. Brasilia: Universidade de Brasília, Brasília; 2009.

33.Torres SECJ, Sousa TCB, Santos AFL, Farias LM, Lima MER. Perfil antropométrico e consumo alimentar de pacientes renais crônicos em hemodiálise. BRASPEN J 2018; 33(4):370-8.

34. Nunes RR, Clemente ELS, Pandini JA, Cobas RA, DiasVM, Sperandei S, et al. Confiabilidade da classificação do estado nutricional obtida através do IMC e três diferentes métodos de percentual de gordura corporal em pacientes com diabetes melito tipo 1. Arq Bras Endocrinol Metab. 2009; 53(3):360-7. http://doi.org/10.1590/50004$\underline{27302009000300011}$

35.Cruz-Jentoft AJ, Baeyens JP, Bauer JM, Boirie $Y$, Cederholm T, Landi $F$, et al. Sarcopenia: European consensus on definition and diagnosis. Age Ageing. 2010; 39(4):412-23. https://doi.org/10.1093/ageing/afq034

36.Kopple JD. National Kidney Foundation K/DOQI Clinical Practice Guidelines for Nutrition in Chronic Renal Failure. 2001; 37(1-supp2):S6670. https://doi.org/10.1053/ajkd.2001.20748

37.Nerbass FB, Cuppari L, Avesani CM, Kamimura MA. Nutrição na doença renal crônica. Barueri: Manole; 2013. p.247-69.

38.Sanches APLM. Doença renal crônica e recomendações nutricionais de macronutrientes. Monografia (Programa de Aprimoramento 
Profissional/SES- Hospital das Clínicas de Ribeirão Preto), Ribeirão Preto, 2008.

39. Oliveira CMC, Kubrusly M, Mota RS, Silva CAB, Oliveira VN. Desnutrição na insuficiência renal crônica: qual o melhor método diagnóstico na prática clínica?. J Bras Nefrol. 2010; 32(1):57$70 . \quad$ https://doi.org/10.1590/S0101$\underline{28002010000100011}$

40. Ribeiro $M$, Araújo ML, Cunha L, Ribeiro D, Pena G. Análise de diferentes métodos de avaliação do estado nutricional de pacientes em hemodiálise. Rev Cuid. 2015; 6(1):932-40. http://doi.org/10.15649/cuidarte.v6i1.163

41.Guedes ERA. Importância do Exercício Físico em Idosos com Sarcopenia. [Monografia] Belo Horizonte: Universidade Federal de Minas Gerais; 2019.

42.Martins C, Cuppari L, Avesani C, Gusmão MG. Projeto Diretrizes: terapia nutricional para pacientes em hemodiálise crônica. 2011.

43. Luz CA, Cortes ML, Souza JS, Gomes LFO, Alves $A B$, Cairo IG. Avaliação da ingestão nutricional de pacientes com doença renal crônica em tratamento hemodialítico. BRASPEN J. 2017; 32(3):241-5.

44.Sociedade Brasileira de Nefrologia. Diretrizes brasileiras de prática clínica para distúrbio mineral e ósseo na doença renal crônica. Jornal Brasileiro de Nefrologia. 2011; 33(2):189-247.

45.Abensur H. Deficiência de ferro na doença renal crônica. Rev Bras Hematol. 2010; 32(2):95$8 . \quad$ http://doi.org/10.1590/S1516$\underline{84842010005000047}$

46. Abensur H. Anemia na doença renal crônica. B J Neprhology. 2008; 24(3):26-8.

47.Fouque DC, Vennegoor M, Wee PT, Wanner C, Basci A, Canaud B, et al. EBPG Guideline on nutrition. Nephrol Dial Transplant. 2007; 22(Suppl 2):45-87. https://doi.org/10.1093/ndt/gfm020

48. Koehnlein EA, Nunes AY, Bordini ACG. Avaliação do estado nutricional de pacientes em hemodiálise. Acta Scientiarum. 2008; 30(1):65-71. https://doi.org/10.4025/actascihealthsci.v30i1.43

97

Colloq Vitae 2020 set-dez; 12(3): 16-25.

Artigo Open Access sob uma licença CC BY-NC-ND (http://creativecommons.org/licenses/by-nc-nd/4.0/). 